\title{
Abortion, Stile Scary Obsession
}

\author{
Mazin S Salman* \\ PHD Medical Microbiology, Ministry of Education, Iraq \\ *Corresponding Author: Mazin S Salman, PHD Medical Microbiology, Ministry of Education, Iraq.
}

Received: October 21, 2019; Published: October 28, 2019

DOI: 10.31080/ASMI.2019.02.0414

Abortion is an important medical problem, in addition to its health risks, it causes psychological, economic and family problems for aborted women, and is an important event affecting women of childbearing age. Miscarriage often occurs spontaneously called spontaneous abortion, may occur early before the twelfth week of pregnancy and is called early spontaneous abortion, or may occur during weeks 20 - 22 of pregnancy and then called late spontaneous abortion [1]. A one-time abortion may be called a single spontaneous abortion or it may happen multiple times and is called recurrent spontaneous abortion, which accounts for 15$20 \%$ of clinically distinct pregnancies [2].There are many reasons for abortion, including genetic factors, which account for more than $5 \%$ of recurrent spontaneous abortions, which are chromosomal abnormalities in one or both spouses and hormonal factors that make up $10-15 \%$ of cases of recurrent spontaneous abortion, which is a lack of certain hormones such as progesterone responsible for the maintenance of pregnancy, as well as in the case of the mother's diabetes uncontrolled, which raises the risk of miscarriage and congenital malformations of fetuses by $12.3 \%$ [3]. Anatomical factors account for $10-15 \%$ of recurrent and spontaneous abortions Congenital dysfunction of the uterine septum, intrauterine adhesions and cervical relaxation. Other causes of abortion are infectious agents, which account for $3-20 \%$ of abortions [4]. Common organisms that cause fetal-placental infections, which are cellular viruses such as CMV and HIV, as well as primary cellular parasites such as Toxoplasma gondii and bacterial cellular pathogens such as Listeria. autoimmune diseases such as lupus erythematosus and phospholipid antibody syndrome represent of the causes of recurrent spontaneous abortions related to the mother's immune system of the mother, including. Immune dysfunctions account for about $60 \%$ of recurrent spontaneous abortions due to an immunological mismatch in the mother's body because the antigens (Alloantigens) of the fetus encode genes inherited from the father that provoke cellular immune reactions in the mother leading to fetal loss and there is evidence that pregnancy leads to suppression Cellular immunity in the mother in order to prevent Fetal rejection. Maternal tissue rejects the developing fetal tissues and trophoblasts as foreign bodies to the maternal immune system [5]. Repeated abortion in women have returned to be a real problem that overlaps with many contemporary problems, especially the problem of antibiotic resistance by microorganisms and the emergence of more deadly viruses.

\section{Bibliography}

1. Cubo-Nava A., et al. "Medical versus surgical treatment of first trimester spontaneous abortion: A cost-minimization analysis". PLoS ONE 14 (2019): e0210449.

2. Nigro G., et al. "Role of the infections in recurrent spontaneous abortion". The Journal of Maternal-Fetal and Neonatal Medicine 24.8 (2011): 983-989.

3. Magon N and Kumar P "Hormones in pregnancy". Nigerian Medical Journal 53.4 (2012): 179-183.

4. Jones RK., et al. "Perceptions of Male Knowledge and Support Among U.S. Women Obtaining Abortions". Women's Health Issues 21 (2011): 117-123.

5. Ziemba-Goldfarb A. "Possible Mechanisms That Protect the Fetus from Maternal". The Science Journal of the Lander College of Arts and Sciences 10 (2017): 56-61.

Volume 2 Issue 11 November 2019

(C) All rights are reserved by Mazin S Salman. 